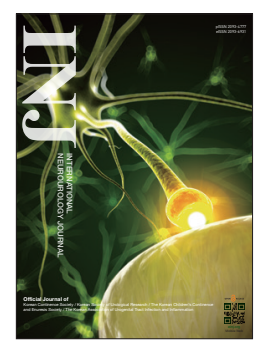

\title{
The Current Positioning of Augmentation Enterocystoplasty in the Treatment for Neurogenic Bladder
}

\author{
Seong Jin Jeong ${ }^{1}$, Seung-June $\mathrm{Oh}^{2}$ \\ ${ }^{1}$ Department of Urology, Seoul National University Bundang Hospital, Seongnam, Korea \\ ${ }^{2}$ Department of Urology, Seoul National University Hospital, Seoul National University College of Medicine, Seoul, Korea
}

\begin{abstract}
Augmentation enterocystoplasty (AEC) is a surgical procedure in which the bladder is enlarged using an intestinal segment in patients with lower urinary tract dysfunction who fail to achieve satisfactory results with all conservative treatments. Currently, surgical materials and procedures, concomitant correction of upper urinary tract abnormalities, or bladder neck reconstruction may vary depending on the experience and preferences of the surgeons. AEC has been proven to be successful with respect to surgical goals, such as achieving urinary continence, improving quality of life, and preserving the upper urinary tract over the long term. The advantage of AEC over intravesical injection of botulinum toxin - a more recent and less invasive procedure - is that the prevention of upper urinary tract damage and the improvement of urinary incontinence are more reliably guaranteed, especially considering that these surgical effects are permanent. Compared to less invasive treatments, the quality of life of patients after surgery is also much higher, and AEC may be more cost-effective in the long run. Thus, in patients with neurogenic bladder, AEC is still the gold standard surgical procedure with strong evidence in support of its efficacy. In this article, the indications, surgical methods, possible complications, long-term follow-up, and current positioning of AEC in lower urinary tract dysfunction is discussed.
\end{abstract}

Keywords: Urinary bladder; Neurogenic; Reconstructive surgical procedures; Urinary incontinence

- Conflict of Interest: No potential conflict of interest relevant to this article was reported.

\section{INTRODUCTION}

In lower urinary tract dysfunction where the storage function is significantly diminished, the patient's quality of life is significantly reduced. Additionally, kidney damage occurs when the urodynamic properties of the urinary bladder are poor. In this situation, surgical treatments are frequently needed to both improve the patient's quality of life and protect the renal function. Augmentation enterocystoplasty (AEC) or urinary diversion should be considered when maximal conservative treatment fails, when primary lower urinary tract reconstruction fails, and when the upper urinary tract is functionally deteriorated in patients with neurogenic lower urinary tract dysfunction.

Recently, the popularity of lower urinary tract reconstruction using the intestine in patients with lower urinary tract dysfunction has greatly declined. This is mainly due to the fact that effective and less invasive treatments have been relatively successful. In this article, the indications, surgical methods, possible complications, long-term follow-up, and current positioning of AEC in lower urinary tract dysfunction are reviewed and discussed.
Corresponding author: Seung-June Oh (iD https://orcid.org/0000-0002-0322-3539 Department of Urology, Seoul National University College of Medicine, Seoul National University Hospital, 101 Daehak-ro, Jongno-gu, Seoul 03080, Korea E-mail: sjo@snu.ac.kr

Submitted: April 21, 2020 / Accepted after revision: June 14, 2020
This is an Open Access article distributed under the terms of the Creative Commons Attribution Non-Commercial License (https://creativecommons.org/licenses/by-nc/4.0/) which permits unrestricted non-commercial use, distribution, and reproduction in any medium, provided the original work is properly cited. 


\section{HISTORY OF LOWER URINARY TRACT RECONSTRUCTION AND RELATED TERMINOLOGY}

Historically, lower urinary tract reconstruction using the bowel began in Europe. AEC using the ileum dates back to 1899 where the surgery was performed in a patient with bladder exstrophy [1]. However, routine performance of AEC started in the 1950s when Couvelaire [2] performed AEC in tuberculous contracted bladder. Techniques involving bowel detubularization began in the 1950s. In the early 1970s, Lapides et al. [3] introduced clean intermittent catheterization (CIC) to the management of neurogenic bladder. AEC began to be more widely practiced as the CIC method successfully assisted postoperative management of the reconstructed bladder.

The terms describing lower urinary tract reconstruction procedures using the intestine vary widely. Lower urinary tract reconstruction can be largely divided into AEC, bladder substitution, and urinary diversion. In noncontinent urinary diversion, the urine storage device is placed outside the skin, whereas in continent urinary diversion, the urine reservoir is placed within the abdominal cavity. Furthermore, continent urinary diversion can be categorized into continent cutaneous urinary diversion, which diverts urine flow by creating a new urine drainage path, and orthotopic urinary diversion, which connects the reservoir to the urethra, which is the original urine drainage pathway. In some patients undergoing AEC, the native urethra may not be used due to very poor sphincter function. In these cases, continent urinary diversion together with AEC may be required, and a strict distinction between the AEC and the urinary diversion is often difficult. The development of neobladder or orthotopic bladder replacement in bladder cancer has contributed significantly to the development of the methods and techniques applied in AEC surgery in patients with neurogenic bladder.

\section{AIM OF THE AUGMENTATION CYSTOPLASTY}

AEC is a surgical method that corrects the pathologic bladder by remodeling the bladder using an intestinal segment. The primary purpose of the procedure is to reduce intravesical pressure to preserve the kidney. This can also increase urinary bladder capacity, and restore urinary continence. In patients having neurogenic bladder with high intravesical pressure, the wall of the urinary bladder is often thick, stiff, and noncompliant. Therefore, intravesical pressure easily increases even when the bladder is slightly filled, resulting in hydronephrosis or urinary incontinence.

The fundamental concept of AEC surgery in the neurogenic bladder should be emphasized (Fig. 1). AEC is not aimed at simply expanding the size of the bladder, but it is a procedure that fundamentally corrects the physical properties of the bladder. Patients with neurogenic bladder often display a wide variety of urodynamic characteristics. As a result, surgery may be applicable to a number of patients even though they may have large bladder capacities. For example, there are cases where the maximum cystometric capacity is as high as $500 \mathrm{~mL}$ but the bladder compliance is very low. Furthermore, when CIC is performed in patients, urinary incontinence and upper urinary tract abnormalities often occur. In these cases, the urologist may face a dilemma as to whether or not AEC of the bladder should be performed. Even in these cases, AEC is often successful in achieving continence and preserving the upper tract. Therefore, AEC should be recognized as a viable method to improve the physical properties of the bladder and to decrease intravesical pressure during bladder storage, and not just to increase the bladder capacity.

\section{PATIENT SELECTION AND PREOPERATIVE EVALUATION}

The classic indication of AEC includes reduced bladder compliance with a very low bladder capacity. In other words, the main indication of AEC is a lower urinary tract dysfunction that progresses to kidney damage or causes urinary incontinence due to the formation of high intravesical pressure despite all conservative treatments. Underlying conditions may include neurogenic bladder caused by various spinal cord diseases such as myelodysplasia and spinal cord injury. The risk of kidney damage in the future, such as vesicoureteral reflux (VUR) or ureteral obstruction, is high in these conditions. Patients may also suffer from urinary incontinence secondary to detrusor overactivity or reduced bladder compliance.

The patient's history, such as any neurological abnormalities, or previous abdominal surgery, must be obtained. Patients with a history of previous intraperitoneal surgery are more likely to have adhesions in the intestine, thus the risk of surgery increases because the mesenteric vessels may be damaged during surgery. In patients with a history of bowel disease, colonoscopy should be performed before surgery to exclude bowel abnormalities, and computed tomography imaging should be consid- 

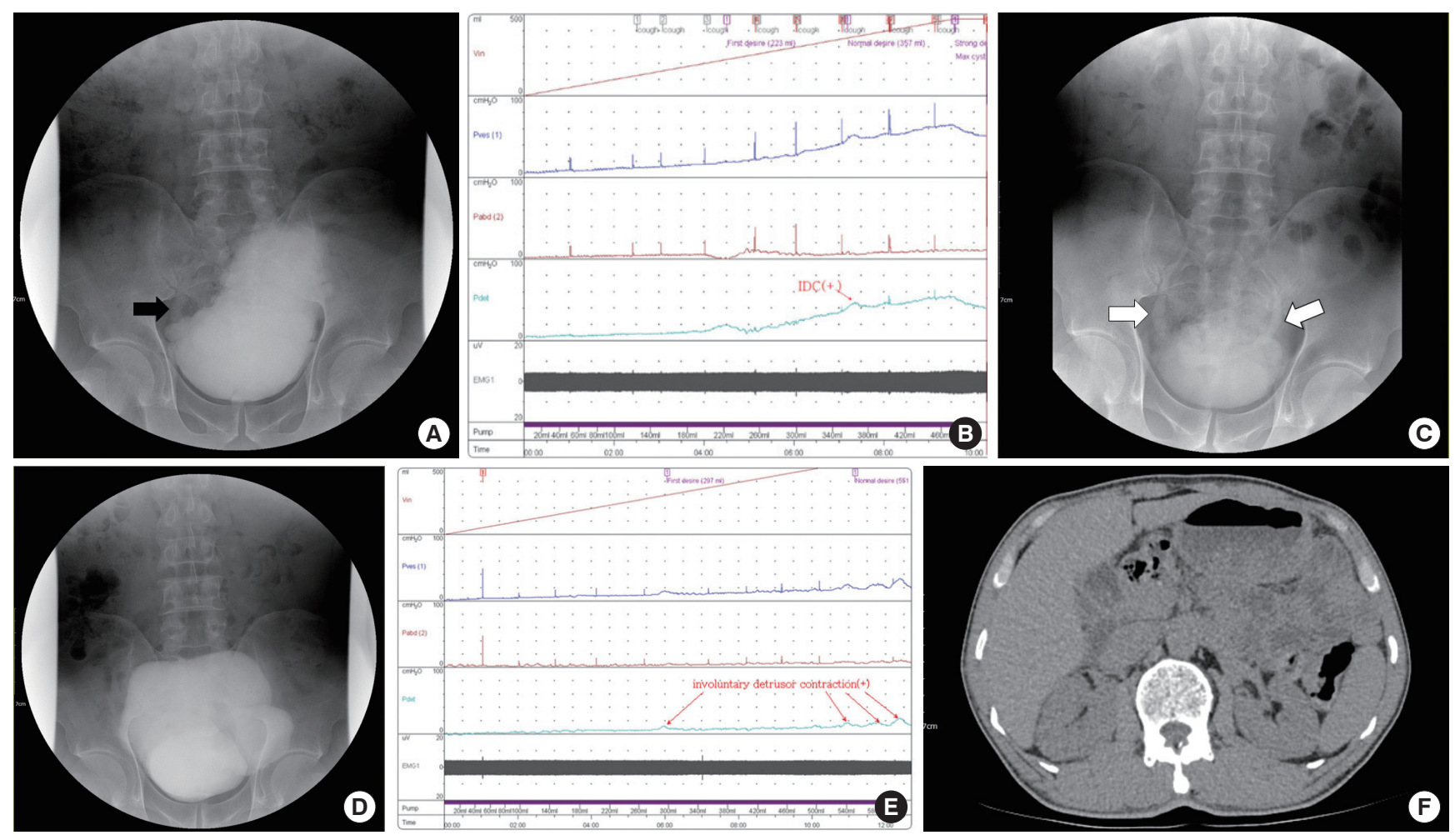

Fig. 1. A 49-year-old male patient with nighttime urinary incontinence together with voiding difficulties following spinal tumor surgery performed 12 years ago. His perineal sensation was decreased and the anal sphincter tone was decreased. (A, B) Fluoroscopic urodynamic study demonstrated that the maximum cystometric capacity (MCC) was $474 \mathrm{~mL}$ and the bladder compliance was very low. The bladder neck was competent, but trabeculation was severe. The right vesicoureteral reflux (VUR) was observed as grade I (black arrow) when the bladder was filled ( $\sim 300 \mathrm{~mL}$ ). (C) Intravenous urography revealed there was no hydronephrosis, but bilateral ureteral columnarization was observed (empty arrows). The ileum $(\sim 40 \mathrm{~cm})$ was harvested and augmentation enterocystoplasty (AEC) surgery without antireflux procedure was performed. In the third week postoperatively, the patient began clean intermittent catheterization 5 times a day. At 11 months, he emptied his bladder up to $600 \mathrm{~mL}$ with an average volume of $400 \mathrm{~mL}$ without experiencing urinary incontinence. (D, E) Urodynamic studies revealed that MCC was $600 \mathrm{~mL}$, detrusor overactivity was weakly observed, and bladder compliance was slightly decreased. (F) However, VUR was not observed and there was no hydronephrosis in noncontrast computed tomography. This case clearly demonstrates that AEC is not simply increase bladder capacity, but plays a fundamental role in correcting the physical properties of the bladder, such as correction of bladder compliance. It also highlights that there is no need for a concomitant antireflux procedure in low-grade VUR.

ered to determine whether there is any abnormality of the bowel. Before surgery, laboratory tests such as renal function tests and serum electrolytes should also be performed. Many patients with lower urinary tract dysfunction who undergo AEC already have renal insufficiency. Therefore, metabolic changes are likely to occur after lower urinary tract reconstruction using the intestine. Prior to surgery, special precautions should be taken to reduce the risk of further decline in the kidney function in patients with renal insufficiency. If AEC is necessary, there should be full disclosure to the patients and their families about the benefits and the potential risks associated with AEC surgery. This will allow patients to have more realistic expecta- tions for the surgery and avoid unnecessary fear.

\section{SELECTION OF THE BOWEL SEGMENT}

In lower urinary tract reconstruction, the selection of the bowel segment is very important. Various procedures have been developed depending on the bowel segment used. The jejunum is rarely used for AEC since it can cause uncorrectable metabolic acidosis and electrolyte imbalance. The most commonly used intestinal segments are the ileum and the ileocecal segment. The ileum has an advantage since it is relatively close to the pelvic area where the bladder is located. It can also provide a lower 
pressure reservoir compared to the large intestine. However, postoperative metabolic acidosis, as well as the malabsorption of bile acid salts and vitamin B12 may occur. Practically, there is not much difference in using an ileocecal segment over using an ileum segment. However, using the ileocecal segment had been advocated for an antireflux purpose as the ileocecal valve could be used as a mechanism to prevent VUR if the ureter is implanted at the terminal ileum. Nevertheless, since loss of the ileocecal valve causes diarrhea, its use is not recommended.

Unfortunately, in many patients who undergo surgery with the ileum, the ileal segment may be unable to reach the bladder trigonal area when the bladder is wide open as the mesenteric artery of the ileum is not long enough. In this case, the colon segment can be a viable alternative. The large intestine has the advantages of being large in diameter, and spatially close to the bladder. However, the large intestine has a disadvantage in terms of the secretion of more mucus than the ileum and metabolic acidosis may also occur. The sigmoid colon is located adjacent to the bladder, so that it can be readily used for bladder reconstruction. However, when using the sigmoid, there is the disadvantage that the urinary continence rate is very low because the bladder capacity is slightly reduced and the pressure in the storage phase is higher.

Seromuscular cystoplasty [4] is designed to reduce metabolic complications, but it is less successful than standard AEC. Today, ileum or sigmoid colon segments are used for lower urinary tract reconstruction, depending on the experience and preferences of the surgeons. If the ileum cannot be used for anatomical or functional reasons, the sigmoid colon is the usual alternative.

\section{SURGICAL TECHNIQUES}

The 2 most important technical principles of the AEC procedure are: (1) the intravesical pressure must be achieved at low pressure, and (2) the reservoir must be geometrically as large as possible with the given limited materials. In order to maintain the low intravesical pressure of the reconstructed bladder, the bowel must be detubularized because the increase in pressure caused by the intrinsic peristaltic movement of the original bowel must be removed. This can be achieved by opening the intestine longitudinally along the antimesenteric border. The intestinal tissue is reconfigured by sewing the detubularized bowel into a $\mathrm{U}$ - or S-shape. Also, in order to maximally increase the bladder capacity, the unfolded bowel must be reconfigured to make it almost spherical. CIC is basically required following AEC [5-7].

The dome of the original bladder should be opened as fully as possible in the longitudinal direction to form a low-pressure storage space. In patients with a severely thickened or fibrous bladder wall, cystectomy may be performed to replace the bladder with the bowel tissue. The reconstructed intestinal tissue is widely anastomosed over the bladder in a water-tight manner. Generally, a suprapubic cystostomy tube is placed in the newly created bladder, separate from the urethral catheter. A drainage tube is also installed around the dependent portion within the abdominal cavity. About 2 weeks after surgery, cystography is performed to confirm that there is no urinary leak in the newly created bladder and the catheters are removed if there is no major leak. Over the course of several months, the bladder volume would rapidly increase and eventually reach around $500 \mathrm{~mL}$. However, the physical properties of the bladder are significantly different than before surgery, so mild existing VUR may disappear. Nonetheless, severe VUR must be surgically corrected simultaneously.

Since the AEC procedure is a relatively major operation, it should be considered to simultaneously correct the existing upper urinary tract or lower urinary tract abnormalities to avoid further surgery. If the bladder neck is severely incompetent, it is highly likely that urinary incontinence will persist after AEC. Therefore, when performing AEC, it may be necessary to perform a bladder neck procedure at the same time. Lee et al. [8] investigated the urinary incontinence after performing AEC only, without performance of bladder outlet procedures in 17 patients who had complained preoperatively of urinary incontinence with an incompetent bladder neck. The International Consultation on Incontinence Modular Questionnaire showed that incontinence disappeared after AEC surgery in $64.7 \%$ of patients. It was found that the rest of the patients also had a statistically significant decrease in the frequency of urinary incontinence. They concluded that concomitant bladder neck surgery is not essential when performing AEC. If necessary, it can be done as a second stage procedure during postoperative followup. Contrary to this report, an interesting study [9] was conducted regarding primary bladder neck reconstruction without AEC. A group of Houston researchers followed 109 pediatric patients with primary bladder neck reconstruction for an average of 8.5 years. At an average of 4.9 years after surgery, $18 \%$ of patients eventually received AEC, $46 \%$ had VUR or hydronephrosis, and $21 \%$ had newly diagnosed or worsening renal scarring. However, the upper urinary tract abnormalities and 
urinary incontinence disappeared in all the patients after AEC. Therefore, the authors concluded that during follow-up the risk of complications was high when only the bladder outlet procedure was performed without AEC, and careful patient followup was essential after the initial surgical decision.

The upper urinary tract abnormality can be largely divided into obstruction and VUR. When intravesical pressure is reduced by using appropriate pharmacotherapy, CIC or a combination of both, then VUR disappears in $\sim 50 \%$ of patients. However, even with these conservative treatments, surgical correction is needed to prevent further kidney damage if upper tract abnormalities persist. Since most VUR is secondary to neurogenic bladder, it disappears as AEC reduces the intravesical pressure (Fig. 1). There is a very interesting observation by Tehran researchers [10] who simply performed AEC without correcting for VUR in 130 patients with neurogenic bladder and VUR. According to their report, VUR was no longer observed in $85.4 \%$, it improved in $50.8 \%$, and it remained or worsened in only $3.8 \%$. Therefore, it is considered that VUR can be successfully treated with AEC only when VUR in patients is accompanied by high intravesical pressure and low bladder compliance.

However, in the author's experience, high grade VUR that persists even at low intravesical pressure requires concomitant correction during surgery. Also, if there is an obstruction at the end of the ureter, the obstructed ureteral portion can be resected and it can be reimplanted into the newly enlarged bladder. The surgeon should predict in advance the method of antireflux mechanism in the neurogenic bladder. Unlike in pediatric primary VUR, the bladder trabeculation is severe and the bladder wall is thick in adult patients with neurogenic bladder, making submucosal tunnels difficult to form and, therefore, the surgical results are often poor. Therefore, when the antireflux procedure is necessary, the author performs ureteral reimplantation on the augmented bowel side rather than on the native bladder. When the colon is used for the AEC, the ureter is reimplanted to the tenia of the colon. If the ileum is used, the antireflux mechanism is successfully achieved by reimplanting the ureter in the ileal chimney by the Studer procedure [11] or by reimplanting the ureter along the extramural intestinal fold by the Ghoneim method [12].

\section{CLINICAL OUTCOMES}

AEC plays a critical role in achieving urinary continence and preventing exacerbation of renal function by fundamentally correcting the physical properties of the bladder. According to a study conducted by Swiss researchers [13], significant improvement in maximum detrusor pressure was achieved from an average of $38 \mathrm{~cm} \mathrm{H}_{2} \mathrm{O}$ preoperatively to $15 \mathrm{~cm} \mathrm{H}_{2} \mathrm{O}$ postoperatively. In addition, bladder capacity was significantly improved from preoperative $(240 \mathrm{~mL})$ to postoperative $(500 \mathrm{~mL})$, and detrusor compliance increased from $13 \mathrm{~mL} / \mathrm{cm} \mathrm{H}_{2} \mathrm{O}$ to $50 \mathrm{~mL} / \mathrm{cm}$ $\mathrm{H}_{2} \mathrm{O}$, respectively. If CIC is properly performed then pyelonephritis caused by high intravesical pressure will not occur. Bacteriuria frequently caused upper urinary tract infections before surgery. But symptomatic urinary tract infections are dramatically reduced postoperatively once VUR is corrected. Longterm follow-up observations revealed that AEC was very successful in preventing deterioration of the upper urinary tract.

Reports investigating patient satisfaction following AEC surgery are very limited. In general, as the high intravesical pressure is corrected after AEC, urinary incontinence due to even borderline sphincter incompetency either disappears or significantly decreases. Since the bladder capacity is significantly increased, the patient's quality of life is dramatically improved because the CIC interval is greatly increased. AEC can greatly assist patients in becoming socially adaptable. The achievement of urinary continence after AEC is reported to be $80 \%$ [14] to $100 \%$ [15]. According to a study by Khastgir et al. [15], the quality of life was reported to be excellent in $96.2 \%$ of patients at an average of 6 months after AEC. According to a report by Shekarriz et al. [16], in the majority of patients $(n=158)$, the continence rate was 95\%. Herschorn and Hewitt [17] investigated surgical outcomes after the AEC procedure in 59 neurogenic bladder patients. They found that all of the patients were satisfied with the operation, and all but one was willing to undergo the AEC surgery again.

Long-term follow-up results after AEC are relatively rare. A French group [18] followed 28 patients with spinal dysplasia (average age 20 years) for an average of 13.6 years. The rate of urinary continence was $71 \%$ where $93 \%$ of patients underwent CIC. Overall complications and reoperation rates were 57\% and $14 \%$, respectively. According to a recent 12 -year long-term follow-up study in 42 pediatric patients conducted by the Saudi Arabian group, it was revealed that electrolyte levels and renal functions were improved or stable in $76.2 \%$, and somatic growth was normal. After AEC surgery, the urinary continence rate was $88.1 \%$, and no bladder perforation or malignancy was found. However, postoperative secondary procedures were re- 
quired in $71.4 \%$ of patients [19]. In many respects, AEC is considered to be very successful after surgery in terms of the urinary continence rate, improvement in quality of life, and longterm preservation of the upper urinary tract.

\section{COMPLICATIONS FOLLOWING AEC}

AEC can have potential short- and long-term complications. Early complications can range from catheter-related problems, infection, urinary leakage, bowel problems, and death. Urinary leakage may occur at the anastomotic site in the bladder immediately after surgery. In addition, urinary leakage may occur if there is an obstruction due to blood clots or mucus in the urinary drainage catheters. Therefore, bladder irrigation with physiological saline is needed periodically from the initial day of surgery. Bowel adhesions or anastomotic stenosis in the bowel can cause intestinal obstruction. One study involving pediatric patients undergoing augmentation ileocystoplasty demonstrated that mid- to long-term complications after surgery were reported at a total of $10 \%$ [20].

Long-term complications included bowel dysfunction (15\%), stone formation (10\%), metabolic abnormalities (3.3\%), and bladder perforation (1.9\%) [21]. According to the Nationwide Readmission Database analysis [22] in a total of 1,873 children under the age of 5, after 18 years of AEC follow-up, the readmission rate was $19.6 \%$ within 30 days after discharge from the hospital. Common reasons for readmission were gastrointestinal complications (19.6\%), urinary tract infection (14.1\%), and wound complications (11.2\%). Therefore, initial complications are not negligible. For this reason, the AEC surgery should be performed in a hospital that has sufficient support systems to manage any complications consequent to the surgery and is able to facilitate life-long follow-up.

One of the most common complications after AEC during long-term follow-up is bladder stones, which is also the most common cause of reoperation after AEC. Risk factors for bladder stone formation include excessive mucus formation, incomplete bladder emptying, noncompliance to CIC or bladder irrigation, bacteriuria caused by urea-splitting organisms. To remove bladder stones, endoscopic or percutaneous approaches, and open surgery can be performed. It is believed that regular irrigation of the augmented bladder may reduce the likelihood of stone formation.

Since a large portion of the bowel segment is permanently incorporated into the urinary tract during AEC surgery, the augmented bladder is constantly exposed to the chemical components of urine. Therefore, it is necessary to consider metabolic changes according to the intrinsic absorption capability of the intestine. Theoretically, as the exposure time of retained urine in the intestinal tissue is prolonged, the acid-base balance or electrolyte balance changes more dramatically. The degree of metabolic change is dependent on the type and length of the bowel segments, the degree of renal or hepatic function, the patient's age, previous history of radiation therapy, and the presence or absence of comorbidities. When AEC is performed with the ileum, postoperative hyperchloremic metabolic acidosis, malabsorption of bile acid salts and vitamin B12 may occur. Hyperchloremic metabolic acidosis may also occur when the colon is used as the material for AEC surgery. Hong Kong researchers performed AEC in 40 adult patients and followed up for $\sim 13$ years [23]. Few complications of renal function and electrolyte imbalance after surgery were reported. There was no significant change in epidermal growth factor receptor (eGFR) after surgery, and postoperative renal function decline was noted in $10 \%$ of patients whose eGFR was less than $24.8 \mathrm{~mL} / \mathrm{min}$ before surgery. Metabolic acidosis occurred in $22.5 \%$ of patients and bicarbonate supplementation was required in $15 \%$ of patients. Taken together, these results suggest that patients with neurogenic bladder who already had impaired renal function before surgery should be closely followed up postoperatively with special attention paid to metabolic imbalances.

Bladder perforation is one of the most serious problems, secondary to peritonitis, sepsis, and even death. Bladder perforation is known to occur in about $8 \%$ of patients after AEC. The causes include chronic bladder overdistention due to poor CIC, chronic infection, trauma during CIC, and ischemic necrosis of long segments. Bladder perforation often requires open exploratory laparotomy.

Controversy exists concerning the development of secondary malignancies after AEC. There is a report that the prevalence of bladder cancer did not increase significantly after AEC using either the small or large intestine (4.6\%) compared to the control group (2.6\%) [24]. The cell types were frequently in the order of adenocarcinoma (52\%), transitional cell carcinoma (39\%), and squamous cell carcinoma (6\%) [25]. The prognosis is known to be relatively poor. The site of tumor development is usually around the suture line between the bladder and intestine. 


\section{POSTOPERATIVE FOLLOW-UP STRATEGY}

Life-long follow-up is essential since surgery-associated morbidity still exists after AEC surgery. Therefore, regular follow-up is needed, especially in patients who have undergone surgery for more than 10 years. Although it has not been ascertained what kind of follow-up evaluation is required, regular follow-up evaluation is recommended as the standard policy. Basic blood chemistry tests should be performed to determine the renal function and metabolic abnormalities. Annual abdominal ultrasonography can be performed to screen for the occurrence of hydronephrosis. Ultrasound and simple radiography are suitable as screening tests for the occurrence of stones in the augmented bladder. There are currently no guidelines instructing on monitoring of the risk of bone mineral reduction due to metabolic changes after AEC. Symptoms of vitamin B12 deficiency can range from very mild to very severe. From the fifth year following AEC, it is recommended to monitor the B12 level annually.

Although it is known that the incidence of malignancy is low, life-long follow-up monitoring for cancer should also be considered. For screening, annual endoscopic examination was previously recommended, but recently it has been found not to be cost-effective. However, patients with hematuria must be evaluated by endoscopy.

\section{COMPARISON BETWEEN BOTULINUM TOXIN AND AEC}

Contraindications to AEC include short bowel syndrome, intrinsic bowel disease such as Crohn disease, and severe kidney disease with a creatinine clearance less than $40 \mathrm{~mL} / \mathrm{min}$, and patients unable to undergo CIC. There are also other groups of patients whose treatment with pharmacotherapy has not been satisfactory or patients who experienced severe drug side effects such as dry mouth, constipation, and blurred vision. In those circumstances, intravesical botulinum toxin (BoT) injection may be considered for further management.

In 2011, the U.S. Food and Drug Administration approved onabotulinum toxin A for intravesical injection therapy for neurogenic detrusor overactivity. Intravesical BoT injection is a minimally invasive and reversible treatment. Cystoscopy is used to inject 200 units of onabotulinum toxin A to the bladder, usually at 10-20 sites. Although there are individual variations, the effect of BoT lasts $\sim 6$ to 12 months in neurogenic bladder pa- tients. CIC is usually required after BoT injection. The urodynamic effects of the intravesical BoT injection are clear. According to a study by Kuo and Liu [26], the maximum detrusor pressure after 4 intravesical injections at 6-month intervals was significantly reduced from $39.8 \mathrm{~cm} \mathrm{H}_{2} \mathrm{O}$ before injection to 20.6 $\mathrm{cm} \mathrm{H}_{2} \mathrm{O}$ after injection. In addition, bladder compliance increased significantly from 26.9 to $40.1 \mathrm{~mL} / \mathrm{cm} \mathrm{H}_{2} \mathrm{O}$.

$\mathrm{AEC}$ is invasive and irreversible, whereas BoT is reversible and less invasive. As a result, it is evident that intravesical injection of BoT has revolutionized the treatment of refractory overactive bladder $(\mathrm{OAB})$. It is a reasonable alternative for patients who are afraid of major surgery like AEC. The AEC, which had previously played a prominent role, has been relegated to a less important position. For example, in the United Kingdom, AEC was conducted in 192 cases in 2000 but it declined to 120 cases in 2010. Interestingly, the number of intravesical BoT injection procedures increased from 50 to 4,088 within the same period [27].

There are few papers that directly compare the effects of BoT injection and AEC in patients with neurogenic bladder. Also, there are limited comparative studies regarding the quality of life issues for each treatment (Table 1). French researchers [28] conducted an interesting comparative study between the 2 treatments in terms of quality of life in patients with refractory $\mathrm{OAB}$ due to spinal cord injury. Quality of life was assessed using the Qualiveen questionnaire [29] in 30 patients who had at least 2 consecutive BoT injections or AEC surgery. The patients in each group were evaluated on average either 9 years after AEC or after undergoing an average of 6.3 BoT injections. Quality of life was significantly lower in the BoT group. The urinary continence rate was significantly higher in the AEC group (complete resolution of urinary incontinence in $87.5 \%$ in the AEC group vs. only $40.3 \%$ in the BoT group). El-Azab and Moeen [30] also analyzed the questionnaire-based assessment of quality of life by dividing 31 patients with refractory idiopathic OAB into BoT and AEC groups. The OAB Satisfaction with Treatment questionnaire score was significantly higher in the AEC group. Patients in the BoT group reported that the need for repeated treatment for symptom control as the primary cause of dissatisfaction. A multicenter prospective study [31] conducted at 7 centers in North America evaluated global bladder function and quality of life in 879 patients. The patients were divided into 3 groups: CIC-only group, BoT-injected CIC group, and AEC-CIC group. The improvement of urinary incontinence and patient satisfaction were the highest in the AEC 


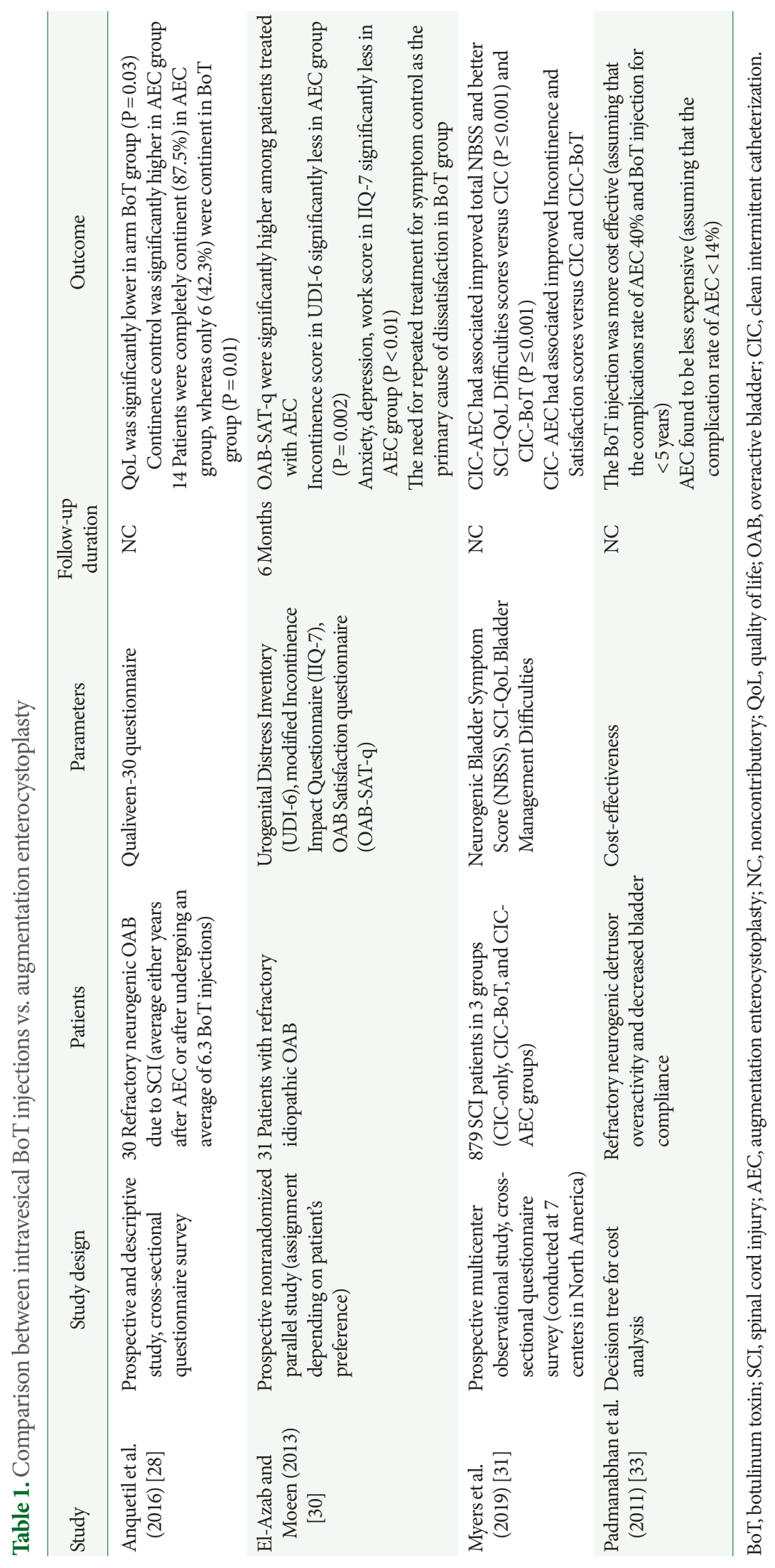


group. The improvement in the global bladder function was significantly higher in older patients, in males, in patients with tetraplegia, and as time after injury increased.

As such, studies demonstrated that the quality of life of neuropathic bladder patients is lower in BoT than in AEC possibly due to the difference in the fundamental nature of the procedures. In other words, AEC is a more effective, reliable, and permanent option for urinary incontinence, resulting in a higher quality of life, while the effect of BoT has an inconsistent duration and variation, leading to poor quality of life. In other words, it is presumed that the inability to predict urinary incontinence between BoT injections and the inability to schedule an injection immediately after urinary incontinence is the most likely cause of deterioration in the quality of life of patients undergoing this procedure.

The long-term risks and benefits of BoT injections should be evaluated in the future. According to the study conducted by Mohee et al. [32], 84 patients decided to discontinue treatment with onabotulinum toxin $\mathrm{A}$. The reasons were recurrent urinary tract infection (23\%), necessity of CIC (49.2\%), inability to perform CIC (9.5\%), and worsening of symptoms despite repeated injections (4.8\%). Padmanabhan et al. [33] performed $\mathrm{AEC}$ and BoT injections for refractory neurogenic bladder and detrusor overactivity, and analyzed the results of follow-up of patients for 5 years from the perspective of cost-effectiveness. The rate of complications arising from AEC was $40 \%$. In this case, The BoT injection was more cost-effective, assuming that the single-time effect of the BoT injection lasted for more than 5.1 months and that treatment was continued for 5 years. However, when the complication rate of AEC is reduced to $14 \%$, the cost of BoT increased and AEC is found to be less expensive. As such, it is also necessary to provide sufficient information to the patient before the procedure and make the patient judge the medico-economic aspect over the long term.

AEC can be successfully applied in very severe inflammatory bladder diseases with reduced bladder capacity and poor bladder compliance due to neurogenic bladder. Intravesical BoT injection is not effective in those conditions due to radiation therapy, chemotherapy, bladder tuberculosis, or interstitial cystitis. Some patients prefer a more permanent solution rather than repeated BoT injections. In addition, it is necessary to consider AEC in neuromuscular diseases such as myasthenia gravis or Guillan-Barre syndrome, which are contraindications to BoT injection. AEC can be a viable choice for the above situations [34]. Currently, AEC is mainly offered to patients with extreme- ly reduced bladder capacity or poor bladder compliance, or bladder that does not respond to or is not suitable for conventional treatment.

The advantage of AEC over BoT injection in neurogenic lower urinary tract dysfunction is that it can improve urinary incontinence and prevent kidney damage. In addition, the improvement is permanent and its effect on quality of life is much higher. It can also be more cost-effective in the long run, for example, over 10 years. In addition, upper urinary tract abnormalities such as VUR and hydronephrosis can be corrected at the same time. Moreover, AEC is still a far more useful option than BoT for some diseases such as refractory interstitial cystitis and tuberculous contracted bladder, where all conservative treatments, including drug therapy, have not been successful [35].

It is assumed that the subjective factors of patients also play a role in the treatment of lower urinary tract dysfunction. For example, when both AEC and BoT bladder injection have been offered to patients as treatment options, patients may naturally prefer BoT injection. Because the BoT injection is well known to the general public and AEC is very unfamiliar to patients, AEC can be difficult to be accepted by the patients. It is also expected that the psychological factors of patients will be considered in the choice of treatment. Patients may generally have a fear of surgery, so they tend to choose a treatment method that is easier to accept even if the outcome is less favorable than that of a large and complication-prone surgery. From personal experience, cultural differences also seem to significantly impact the treatment options. In Korean patients, it is felt that an individual's choice is greatly influenced by the overall opinion of fellow patients, as there is an organized patient association among patients with spinal cord injuries. Regardless of how effective the treatment is, the larger the surgery, the more negative opinions tend to form. Hence, it appears that BoT injection is strongly preferred. In addition, it is believed that various nonmedical factors such as economic cost will greatly influence the choice of treatment.

\section{CONCLUSIONS}

AEC is an established standard surgical method in patients with refractory neurogenic lower urinary tract dysfunction to conventional modalities. AEC can be a very time-consuming, invasive surgery, involving extended hospitalization, with a high risk of potential complications. However, the procedure is 
highly effective and offers a permanent solution for patients with appropriate indications. Even with less invasive modalities, such as intravesical injection of BoT, AEC is still a viable option for storage dysfunction since it is cost-effective in the long run and the quality of life and patient satisfaction are still higher than BoT injection.

\section{ACKNOWLEDGMENTS}

Ms. Hee Jung Kim assisted management of references. Ms. Eugene $\mathrm{Oh}$ aided in proofreading the manuscript.

\section{AUTHOR CONTRIBUTION STATEMENT}

- Conceptualization: $S J O$

- Formal Analysis: SJO, SJJ

$\cdot$ Investigation: $S J O, S J J$

- Methodology: SJO, SJJ

- Project Administration: SJO

- Writing-Original Draft: SJO

-Writing-Review \& Editing: SJO, SJJ

\section{REFERENCES}

1. Rutkowski M. Zur Methode der Harnblasenplastik. Zbl Chir 1899; 16:473-8.

2. Couvelaire R. La petite vessie des tuberculeux genito-urinairses: essai de classification, places et variants des cystointestinoplasties. J Urol Medicale Chir 1950;56:381-434.

3. Lapides J, Diokno AC, Silber SJ, Lowe BS. Clean, intermittent selfcatheterization in the treatment of urinary tract disease. J Urol 1972;107:458-61.

4. Bandi G, Al-Omar O, McLorie GA. Comparison of traditional enterocystoplasty and seromuscular colocystoplasty lined with urothelium. J Pediatr Urol 2007;3:484-9.

5. Cameron AP, Cespedes RD, McGuire EJ. Bladder augmentation. In: Graham Jr. SD, Keane TE, editors. Glenn's urologic surgery. 7th ed. Philadelphia (PA): Wolters Kluwer; 2010. p. 118-24.

6. Cartwright PC. Ileocystoplasty. In: Smith JA, Howards SA, Preminger GM, Dmochowski RR, editors. Hinman's atlas of urologic surgery. 4th ed. Philadelphia (PA): Elsevier; 2018. p. 461-4.

7. Dahl DM. Use of intestinal segments in urinary diversion. In: Wein AJ, Kavoussi LR, Partin, Alan W, Peters CA, editors. CampbellWalsh urology. 11th ed. Philadelphia (PA): Elsevier 2016. p. 2281316.

8. Lee HE, Bae J, Oh JK, Oh SJ. Is concomitant bladder neck recon- struction necessary in neurogenic incontinent patients who undergo augmentation cystoplasty? Korean J Urol 2013;54:42-7.

9. Grimsby GM, Menon V, Schlomer BJ, Baker LA, Adams R, Gargollo PC, et al. Long-term outcomes of bladder neck reconstruction without augmentation cystoplasty in children. J Urol 2016; 195:155-61.

10. Simforoosh N, Tabibi A, Basiri A, Noorbala MH, Danesh AD, Ijadi A. Is ureteral reimplantation necessary during augmentation cystoplasty in patients with neurogenic bladder and vesicoureteral reflux? J Urol 2002;168(4 Pt 1):1439-41.

11. Studer UE, Ackermann D, Casanova GA, Zingg EJ. Three years' experience with an ileal low pressure bladder substitute. $\mathrm{Br} J$ Urol 1989;63:43-52.

12. Abol-Enein H, Ghoneim MA. Further clinical experience with the ileal W-neobladder and a serous-lined extramural tunnel for orthotopic substitution. Br J Urol 1995;76:558-64.

13. Krebs J, Bartel P, Pannek J. Functional outcome of supratrigonal cystectomy and augmentation ileocystoplasty in adult patients with refractory neurogenic lower urinary tract dysfunction. Neurourol Urodyn 2016;35:260-6.

14. Medel R, Ruarte AC, Herrera M, Castera R, Podesta ML. Urinary continence outcomes after augmentation ileocystoplasty as a single surgical procedure in patients with myelodysplasia. J Urol 2002; 168:1849-52.

15. Khastgir J, Hamid R, Arya M, Shah N, Shah PJ. Surgical and patient reported outcomes of 'clam' augmentation ileocystoplasty in spinal cord injured patients. Eur Urol 2003;43:263-9.

16. Shekarriz B, Upadhyay J, Demirbilek S, Barthold JS, González R. Surgical complications of bladder augmentation: comparison between various enterocystoplasties in 133 patients. Urology 2000; 55:123-8.

17. Herschorn S, Hewitt RJ. Patient perspective of long-term outcome of augmentation cystoplasty for neurogenic bladder. Urology 1998;52:672-8.

18. Perrouin-Verbe MA, Léon P, Denys P, Mongiat-Artus P, ChartierKastler E, Phé V. Long-term functional outcomes of augmentation cystoplasty in adult spina bifida patients: a single-center experience in a multidisciplinary team. Neurourol Urodyn 2019;38:330-7.

19. Mehmood S, Alhazmi H, Al-Shayie M, Althobity A, Alshammari A, Altaweel WM, et al. Long-term outcomes of augmentation cystoplasty in a pediatric population with refractory bladder dysfunction: a 12-year follow-up experience at single center. Int Neurourol J 2018;22:287-94.

20. Krishna A, Gough DC, Fishwick J, Bruce J. Ileocystoplasty in children: assessing safety and success. Eur Urol 1995;27:62-6. 
21. Hoen L', Ecclestone H, Blok BFM, Karsenty G, Phé V, Bossier R, et al. Long-term effectiveness and complication rates of bladder augmentation in patients with neurogenic bladder dysfunction: a systematic review. Neurourol Urodyn 2017;36:1685-702.

22. Maldonado N, Michel J, Barnes K. Thirty-day hospital readmissions after augmentation cystoplasty: a nationwide readmissions database analysis. J Pediatr Urol 2018;14:533.e1-533.e9.

23. Cheng KC, Kan CF, Chu PS, Man CW, Wong BT, Ho LY, et al. Augmentation cystoplasty: urodynamic and metabolic outcomes at 10year follow-up. Int J Urol 2015;22:1149-54.

24. Higuchi TT, Granberg CF, Fox JA, Husmann DA. Augmentation cystoplasty and risk of neoplasia: fact, fiction and controversy. J Urol 2010;184:2492-6.

25. Soergel TM, Cain MP, Misseri R, Gardner TA, Koch MO, Rink RC. Transitional cell carcinoma of the bladder following augmentation cystoplasty for the neuropathic bladder. J Urol 2004;172:1649-51.

26. Kuo HC, Liu SH. Effect of repeated detrusor onabotulinumtoxinA injections on bladder and renal function in patients with chronic spinal cord injuries. Neurourol Urodyn 2011;30:1541-5.

27. Biers SM, Venn SN, Greenwell TJ. The past, present and future of augmentation cystoplasty. BJU Int 2012;109:1280-93.

28. Anquetil C, Abdelhamid S, Gelis A, Fattal C. Botulinum toxin therapy for neurogenic detrusor hyperactivity versus augmentation enterocystoplasty: impact on the quality of life of patients with SCI. Spinal Cord 2016;54:1031-5.
29. Costa P, Perrouin-Verbe B, Colvez A, Didier J, Marquis P, Marrel A, et al. Quality of life in spinal cord injury patients with urinary difficulties. Development and validation of qualiveen. Eur Urol 2001;39:107-13.

30. El-Azab AS, Moeen AM. The satisfaction of patients with refractory idiopathic overactive bladder with onabotulinumtoxinA and augmentation cystoplasty. Arab J Urol 2013;11:344-9.

31. Myers JB, Lenherr SM, Stoffel JT, Elliott SP, Presson AP, Zhang C, et al. The effects of augmentation cystoplasty and botulinum toxin injection on patient-reported bladder function and quality of life among individuals with spinal cord injury performing clean intermittent catheterization. Neurourol Urodyn 2019;38:285-94.

32. Mohee A, Khan A, Harris N, Eardley I. Long-term outcome of the use of intravesical botulinum toxin for the treatment of overactive bladder (OAB). BJU Int 2013;111:106-13.

33. Padmanabhan P, Scarpero HM, Milam DF, Dmochowski RR, Penson DF. Five-year cost analysis of intra-detrusor injection of botulinum toxin type A and augmentation cystoplasty for refractory neurogenic detrusor overa ctivity. World J Urol 2011;29:51-7.

34. Shreck E, Gioia K, Lucioni A. Indications for augmentation cystoplasty in the era of onabotulinumtoxinA. Curr Urol Rep 2016;17:27.

35. Wu SY, Jiang YH, Kuo HC. Long-term outcomes of augmentation enterocystoplasty in patients with end-stage bladder diseases: a single-institute experience involving 102 patients. Int Neurourol J 2017;21:133-8. 